\title{
Genetic Algorithm Assisted Joint Multiuser Symbol Detection and Fading Channel Estimation for Synchronous CDMA Systems
}

\author{
Kai Yen and Lajos Hanzo, Senior Member, IEEE
}

\begin{abstract}
A novel multiuser code division multiple access (CDMA) receiver based on genetic algorithms is considered, which jointly estimates the transmitted symbols and fading channel coefficients of all the users. Using exhaustive search, the maximum likelihood (ML) receiver in synchronous CDMA systems has a computational complexity that is exponentially increasing with the number of users and, hence, is not a viable detection solution. Genetic algorithms (GAs) are well known for their robustness in solving complex optimization problems. Based on the ML rule, GAs are developed in order to jointly estimate the users' channel impulse response coefficients as well as the differentially encoded transmitted bit sequences on the basis of the statistics provided by a bank of matched filters at the receiver. Using computer simulations, we showed that the proposed receiver can achieve a near-optimum bit-error-rate (BER) performance upon assuming perfect channel estimation at a significantly lower computational complexity than that required by the ML optimum multiuser detector. Furthermore, channel estimation can be performed jointly with symbol detection without incurring any additional computational complexity and without requiring training symbols. Hence, our proposed joint channel estimator and symbol detector is capable of offering a higher throughput and a shorter detection delay than that of explicitly trained CDMA multiuser detectors.
\end{abstract}

Index Terms - Channel estimation, code division multiple access, genetic algorithms, joint data, multiuser detection.

\section{INTRODUCTION}

C ODE DIVISION multiple access (CDMA) [1]-[4] constitutes an attractive multiuser scheme that allows users to transmit at the same carrier frequency in an uncoordinated manner. However, this creates multiple access interference (MAI), which - if not controlled - can seriously deteriorate the quality of reception. Numerous methods have been proposed for reducing the amount of MAI present in the received signal, such as power control, the optimization of signature sequences, and sectorized antennas. Nevertheless, these techniques have their limitations in combating the effects of MAI, in conjunction with the conventional single user detector, since the MAI is treated as noise.

On the other hand, the so-called multiuser detector [5] treats the MAI as a part of the information, rather than noise. Hence, by processing this additional information, significant performance

Manuscript received April 26, 2000; revised November 17, 2000.

The authors are with the Department of Electronics and Computer Science, University of Southampton, SO17 1BJ, U.K. (e-mail: ky97r@ecs.soton.ac.uk 1h@ecs.soton.ac.uk).

Publisher Item Identifier S 0733-8716(01)01913-8. improvements can be achieved, approaching the ultimate limit of the single-user bound. The optimum multiuser detector [6] based on the maximum likelihood (ML) rule has a computational complexity that is exponentially increasing with the number of users. Hence, the optimum ML multiuser detector is impractical to implement. This complexity constraint led to numerous so-called suboptimal multiuser detection proposals, highlighted in [5] and the references therein, which sacrifice performance for the sake of a reduced complexity. In particular, the reduced complexity tree-search type algorithms [7]-[9] based on the ML rule with a specific number of surviving paths were shown to achieve a nearoptimum performance, when used in conjunction with a noise whitening filter. However, some form of memory is required at the receiver in order to store the metrics of the surviving paths. Conventionally, the fading channel impulse response (CIR) coefficients are usually estimated using a pilot signal [10] as, for example, on the downlink of the IS-95 system [11] in order to facilitate coherent detection. However, this technique becomes inefficient on the uplink, since an independent pilot signal is required from each user in order to estimate the independent fading CIR coefficients experienced by each user's signal. Nonetheless, in order to support multiuser detection, this approach was used in the third-generation UTRA system [4]. The associated inefficiency can be eliminated by invoking joint channel and data estimation, which is the topic of this paper.

Recently, the notion of joint multiuser symbol detection and channel estimation was addressed in [7], [12]-[14], and [16]. In [7], symbol detection is accomplished using a tree-search algorithm, while the users' complex signal amplitudes are estimated using recursive least-squares techniques. In [12], Gauss-Seidel iterations are applied, in order to solve the joint symbol detection and channel estimation problem. The channel estimation is performed via the expectation maximization (EM) algorithm, while a multistage detection algorithm is used for detecting the data packets. In [13], joint multiuser detection and channel estimation is performed using two types of decorrelators in conjunction with a channel estimator. A path-by-path decorrelator is used to provide noisy channel information for the channel estimator, while a channel-matched decorrelator decides on the symbols transmitted and these decisions are fed back to the channel estimator as reference signals. In [14] and [15], a decorrelator and a Kalman filter were used for symbol detection and channel estimation, respectively. A per-survivor approach was also proposed in [14], which used a bank of Kalman filters for channel estimation. Furthermore, in [16] a multiuser receiver based on the Kalman filter was used for joint symbol detection and channel estimation. 
We note that in all the proposed methods mentioned above [7], [12]-[16], the symbol detection and channel estimation were performed using two separate but interlinked techniques, which potentially incurs additional complexity.

In this paper, we present a novel approach to the problem of joint symbol detection and channel estimation in DS/CDMA over flat-fading channels based on a genetic algorithm (GA) innovation. GAs have been employed for solving many complex optimization problems in numerous fields. While GAs are not perfect, i.e., they do not always find the optimal point, they are very efficient in attaining near-optimal solutions significantly faster than conventional point-by-point exhaustive search techniques, especially in large solution spaces. Based on the ML rule, we developed a GA that jointly estimates all the users' channel coefficients as well as the transmitted bit sequences on the basis of the statistics provided by the bank of matched filters at the receiver. GA-based multiuser detection has been proposed, e.g., in [17]-[20], where the analysis was based on the AWGN channel, where the only variables of interest are the users' transmitted bits. Hence, the search space was discrete with a finite number of search points that is exponentially dependent on the number of users. In this paper, we demonstrate that such a GA-based multiuser detector can also be applied to symbol detection over a fading channel, if the CIR coefficients are known. However, in the context of joint CIR estimation and symbol detection solely by GAs - as considered here-the search space is continuous with an infinite number of possible points, simply because the fading attenuation and phase trajectories are continuous. A GA-based channel estimation technique has been proposed previously in [21], which employed the Viterbi algorithm for data detection in a single-user receiver. Hence, our proposed GA-based multiuser detector is slightly different in terms of its structure from those in [17]-[21]. We will show in Section III that the CIR estimation can be performed jointly with the symbol detection using the same GAs simultaneously, without incurring any additional computational complexity, unlike in [7], [12]-[16]. Furthermore, in contrast to Kalman filter-based CIR estimation [16], which is CIR-dependent, no knowledge of the CIR is required for our proposed estimator. Since the CIR estimation can be conducted without explicit training sequences or decision feedback, our proposed detector is capable of offering a potentially higher throughput and a shorter detection delay than that of explicitly trained CDMA multi-user detectors.

The CIR estimation and symbol detection performance of the proposed multiuser detector is examined by computer simulations. The performance measures of interest in this paper are the mean-squared error (MSE) and the BER for the CIR estimation and symbol detection, respectively. We will show that our proposed receiver can achieve a near-optimum BER performance with the aid of perfect channel estimation at a significantly lower computational complexity than that required by the ML optimum multiuser detector. The MSE performance of the CIR estimator is particularly crucial, since it was shown in [15], [22]-[24] that a high CIR estimation MSE can cause a significant BER degradation, which induces an irreducible error floor.

The paper is organized as follows. Section II describes the system model used in this paper. We consider a synchronous
CDMA system over a single-path frequency-nonselective fading channel. Section III describes the GAs used to implement our proposed joint multiuser channel estimator and symbol detector in the context of slowly fading channels. Our simulation results are presented in Section IV. Section V concludes the paper.

\section{SYSTEM DESCRIPTION}

We consider a symbol-synchronous CDMA system, where $K$ users transmit data packets over a single-path frequency-nonselective slowly Rayleigh fading channel, and hence, no multipath diversity can be exploited. Assuming ideal low-pass filtering, the baseband received signal is given by

$$
r(t)=S(t, \boldsymbol{b})+n(t)
$$

where $n(t)$ is the zero-mean complex additive white Gaussian noise (AWGN) with independent real and imaginary components, each having a double-sided power spectral density of $N_{0} / 2$ and

$$
\begin{aligned}
& S(t, \boldsymbol{b})=\sum_{i=0}^{M-1} \sum_{k=1}^{K} \sqrt{\xi_{k}} c_{k}^{(i)} b_{k}^{(i)} a_{k}\left(t-i T_{b}\right), \\
& 0 \leq t<T_{f} .
\end{aligned}
$$

In (2), $M$ is the number of data bits in a frame transmitted by each user, $\xi_{k}$ is the bit energy of the $k$ th user, $T_{b}$ is the signaling interval, $T_{f}$ is the frame duration, and $a_{k}(t)$ is the signature sequence of the $k$ th user associated with a processing gain of $N_{c}$. However, our proposed detector is capable of handling sequences of long period, as long as the cross-correlations between the users' signature sequences over each signaling interval are known to the detector. The unknown variables in (2) are $b_{k}^{(i)} \in\{+1,-1\}$ and $c_{k}^{(i)}$, which denote the $i$ th bit and the corresponding complex CIR coefficient of the $k$ th user, respectively. The channel is assumed to be slowly fading, such that $c_{k}^{(i)}$ may be taken to be constant over one signaling interval and the fading is independent for all users. It is also assumed that $c_{k}^{(i)}$ varies over the duration $T_{f}$ of the $M$-bit transmission frame according to the Doppler frequency $f_{d}$. There are numerous models that can be used to describe the fading channel characteristics, for example, the Jakes model [5] or a first-order Gauss-Markov model as given by [15]

$$
c_{k}^{(i+1)}=a c_{k}^{(i)}+\nu_{k}^{(i)}
$$

where $a=\exp \left(-2 \pi f_{d} T_{b}\right)$ and $\nu_{k}^{(i)}$ is a zero-mean white Gaussian variable. Kalman filter-based CIR estimation requires exact knowledge of $a$ and that of the variance of $\nu_{k}^{(i)}$, which must be acquired with the aid of training symbols and updated frequently over a time-variant channel [24]. Alternatively, we can express the relation between $c_{k}^{(i)}$ and $c_{k}^{(i+1)}$ as

$$
c_{k}^{(i+1)}=c_{k}^{(i)}+\Delta_{k}^{(i)}
$$

where $\Delta_{k}^{(i)}$ is a random variable whose value is dependent on $a=\exp \left(-2 \pi f_{d} T_{b}\right)$ and $\nu_{k}^{(i)}$. Note that (4) is analogous to the 


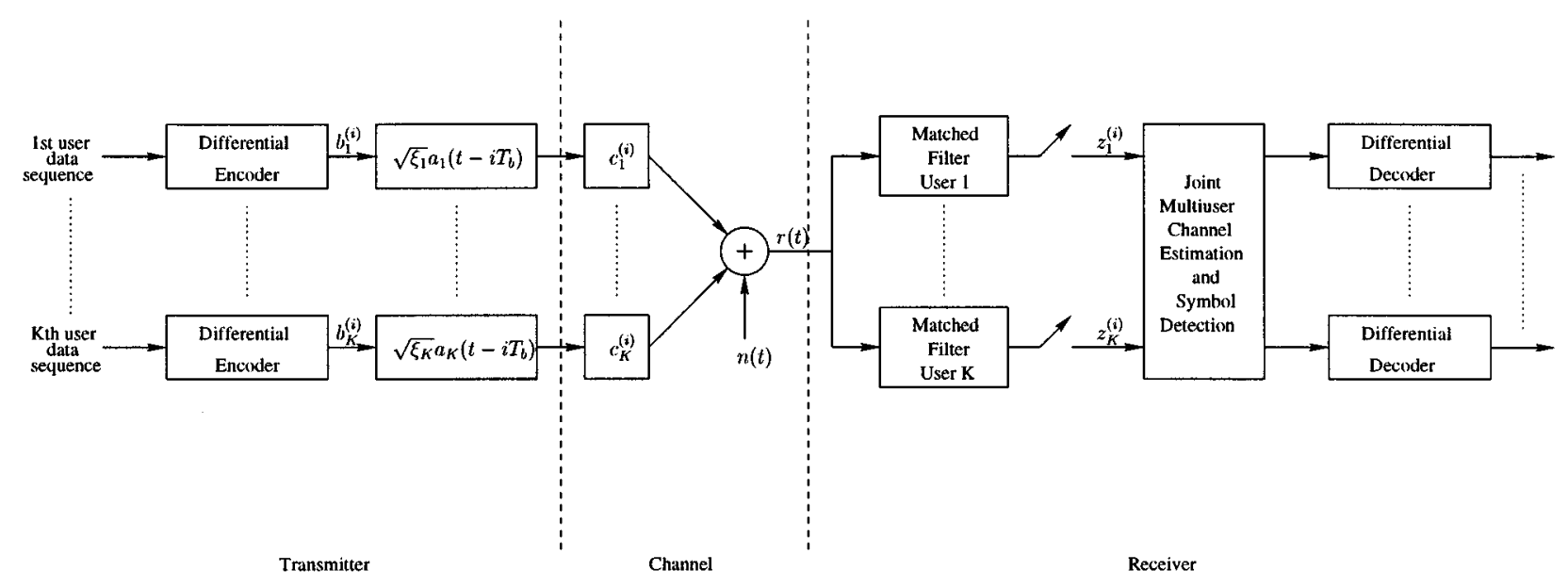

Fig. 1. Block diagram of the system model.

LMS-based CIR estimator, in which $\Delta_{k}^{(i)}=\mu b_{k}^{(i)^{*}}\left[c_{k}^{(i)} b_{k}^{(i)}+\right.$ $\left.n_{k}^{(i)}-\hat{c}_{k}^{(i)} b_{k}^{(i)}\right]$ (see [25, eq. (8)]), where $\mu$ is the step size.

In the context of joint channel estimation and symbol detection using phase shift keying (PSK), there is always the inherent problem of a phase ambiguity of $\pi$ in the estimated CIR coefficients. In order to overcome this problem, the transmitted bit sequence is differentially encoded [22]. Our system model is shown in Fig. 1.

At the receiver, a bank of filters matched to the corresponding set of the users' signature sequences is sampled at the end of the $i$ th bit interval. In this paper, we are interested in determining the unknown data bits $b_{k}^{(i)}$ for $i=1, \ldots, M-1$ and $k=1, \ldots, K$ as well as the CIR coefficients $c_{k}^{(i)}$ for $k=1, \ldots, K$ and $i=$ $0, \ldots, M-1$ at the receiver, in order to perform coherent detection of the received signals. It is convenient to formulate the output $z$ of the matched filters in vector notation, which is given by

$$
z=\left[z_{1}^{(i)}, \ldots, z_{K}^{(i)}\right]^{T}=\boldsymbol{R} \boldsymbol{C}^{(i)} \boldsymbol{\xi}^{(i)}+\boldsymbol{n}
$$

where $\boldsymbol{R}$ is a $K \times K$ user signature sequence cross-correlation matrix with elements given by

$$
\begin{gathered}
R_{i j}=\int_{0}^{T_{b}} a_{i}(t) a_{j}(t) d t \\
\boldsymbol{C}^{(i)}=\operatorname{diag}\left[c_{1}^{(i)}, c_{2}^{(i)}, \ldots, c_{K}^{(i)}\right] \\
\xi=\operatorname{diag}\left[\sqrt{\xi_{1}}, \sqrt{\xi_{2}}, \ldots, \sqrt{\xi_{K}}\right] \\
\boldsymbol{b}^{(i)}=\left[b_{1}^{(i)}, b_{2}^{(i)}, \ldots, b_{K}^{(i)}\right]^{T}
\end{gathered}
$$

and

$$
\boldsymbol{n}=\left[n_{1}^{(i)}, n_{2}^{(i)}, \ldots, n_{K}^{(i)}\right]
$$

is a zero mean Gaussian noise vector with a covariance matrix $R_{n}=0.5 N_{0} \boldsymbol{R}$.

Based on the observation vector $z$ given in (5), it can be shown that the so-called log-likelihood function (LLF) conditioned on the matrix $C^{(i)}$ containing the CIR coefficients in its diagonal and on the vector $\boldsymbol{b}^{(i)}$ incorporating the data bits is given by [8], [12]

$$
\begin{aligned}
\Lambda\left(\boldsymbol{C}^{(i)}, \boldsymbol{b}^{(i)}\right)= & 2 \Re\left\{\boldsymbol{b}^{(i)^{T}} \xi\left(\boldsymbol{C}^{(i)}\right)^{*} \boldsymbol{z}\right\} \\
& -\left(\boldsymbol{b}^{(i)}\right)^{T} \xi \boldsymbol{C}^{(i)} \boldsymbol{R}\left(C^{(i)}\right)^{*} \boldsymbol{\xi} \boldsymbol{b}^{(i)}
\end{aligned}
$$

where $(\cdot)^{*}$ denotes the conjugate operation. The decision rule for the optimal joint multiuser channel estimation and symbol detection scheme is to choose the channel coefficient matrix $\hat{\boldsymbol{C}}^{(i)}$ and symbol vector $\hat{\boldsymbol{b}}^{(i)}$, which maximizes the LLF given in (7) under a constraint on the channel coefficient matrix $C^{(i)}$ as imposed by (4). Hence,

$$
\begin{aligned}
\left(\hat{\boldsymbol{C}}^{(i)}, \hat{\boldsymbol{b}}^{(i)}\right)=\arg \left\{\max _{\boldsymbol{C}^{(i)}, \boldsymbol{b}^{(i)}}\left[\Lambda\left(\boldsymbol{C}^{(i)}, \boldsymbol{b}^{(i)}\right)\right]\right\} \\
\quad \text { subject to } \boldsymbol{C}^{(i)}=\boldsymbol{C}^{(i-1)}+\boldsymbol{\Delta}^{(i-1)} .
\end{aligned}
$$

Equation (8) constitutes a global optimization problem and is nonlinear, since it entails taking the maximum of $\Lambda\left(\boldsymbol{C}^{(i)}, \boldsymbol{b}^{(i)}\right)$. Hence, (8) cannot be solved by a conventional linear optimization approach or by an exhaustive tree search, because the actual values of the channel coefficient matrix $C^{(i)}$ in (7) are unknown, unless a separate CIR estimator is incorporated, as in [7].

\section{Genetic Algorithm Based Joint Channel Estimation AND SYMBOL DETECTION}

The efficiency of any global optimization technique can be measured in terms of two properties: the so-called explorative property and the exploitative property [26]. Techniques that possess a high explorative property have a slower convergence rate and a higher computation complexity but they explore the entire space in order to locate the global optimum. Hence, accuracy is always guaranteed. The optimal ML multiuser detector [6], which computes the LLF for every point in the solution space, is an example of such techniques. On the other hand, techniques such as the family of hill-climbing methods possess a high exploitative property, and hence they offer fast convergence to an optimum of a given subspace. However, this optimum may not be the global optimum of the entire solution space. Unless that particular subspace contains the global optimum, the outcome will always be suboptimum, resulting in a so-called premature 
convergence. For example, the tree-search techniques based on the $M$-algorithm [8] retaining a limited number of surviving paths can be said to favor exploitation rather than exploration, since once a trellis path is discarded, it will not be reconsidered.

GAs [27]-[29] constitute robust global search and optimization strategies that can strike an attractive balance between exploitation and exploration. These algorithms were introduced by Holland [27], and their principles are based on the concept of natural evolution. Specifically, GAs use a population of candidate solutions initially distributed randomly over the entire solution space. Hence, GAs are highly explorative at the beginning. By evolving this population of candidate solutions over successive iterations or generations, through probabilistic transition operations based on Darwinian survival of the fittest, the GA quickly identifies and exploits the subspaces, in which the global optimum may be located, while at the same time maintains the exploration of other parts of the solution space. Hence, while the optimum solution is not always located, the GA has a low probability of curtailing the exploration in suboptimal, rather than optimal solutions. For an in-depth examination of the principles of GAs [27]-[29] are highly recommended. In this paper, we employ GAs in order to solve the joint CIR estimation and symbol detection optimization problem, where the required objective function is defined by the LLF in (7). Specifically, we are interested in determining the CIR matrix $\hat{\boldsymbol{C}}^{(i)}$ and the data vector $\hat{\boldsymbol{b}}^{(i)}$ that maximize this objective function. The structure of the proposed GA-based CIR estimator and symbol detector can be best understood with the aid of the flowchart shown in Fig. 2, which will be referred to often.

\section{A. Initialization}

Initialization of the GA is performed at the so-called $(y=1)$ st generation for each new signaling interval, as seen in Fig. 2, by creating $P$ number of candidate solutions, or strings in GA parlance. The set of $P$ strings is known as a population, and $P$ is known as the population size. These strings represent the unknown variables of interest, which in this case are the diagonal CIR matrix $C^{(i)}$ and the data vector $\boldsymbol{b}^{(i)}$ of (7). Hence, each string will contain $K$ elements corresponding to the $K$ users, each assuming a certain value. We shall denote the complex-valued CIR coefficient strings as $\tilde{\boldsymbol{C}}_{p}^{(i)}(y)=\left[\tilde{c}_{p, 1}^{(i)}(y), \tilde{c}_{p, 2}^{(i)}(y), \ldots, \tilde{c}_{p, K}^{(i)}(y)\right]$, which is composed of real and imaginary parts, and the data string as $\tilde{\boldsymbol{b}}_{p}^{(i)}(y)=\left[\tilde{b}_{p, 1}^{(i)}(y), \tilde{b}_{p, 2}^{(i)}(y), \ldots, \tilde{b}_{p, K}^{(i)}(y)\right]$, which is composed of the antipodal bits of the $K$ users at instant $i$. Explicitly, the parameters $i, y$, and $p$ denote the $i$ th signaling interval, the $y$ th generation, and the $p$ th string, respectively. The reason for using two sets of strings - rather than one-is purely to differentiate between the complex CIR coefficient strings $\tilde{C}_{p}^{(i)}(y)$ and the data strings $\tilde{\boldsymbol{b}}_{p}^{(i)}(y)$, since the mutation process of these strings seen in Fig. 2 is different, which will be further elaborated on later in this section. Furthermore, such distinction can easily cater for situations when, e.g., known pilot symbol assisted CIR estimates are available. We then simply assign these $a$ priori known CIR estimates to all the corresponding strings without changing the structure of the GA. In this case, only the data strings will be processed by the GAs. This process is reversed appropriately, if the CIR coefficients are estimated using the GA-based CIR estimator in conjunction with known bits. We shall use the terminology combination string whenever we refer to both the CIR coefficient-string and the data-string. Hence, the $p$ th combination string will consist of the $p$ th CIR coefficient-string and the $p$ th data-string.

In order to attain a highly diversified search (exploration) at the beginning without knowing where the optimum solution may be located, it is desirable to distribute the candidate solutions randomly throughout the solution space. Hence, the elements of the antipodal bit strings $\widetilde{\boldsymbol{b}}_{p}^{(i)}(y)$ for $p=1, \ldots, P$ and $i=1, \ldots, M-1$ at $y=1$ are randomly generated +1 or -1 values with equal probability. Similarly at $i=0$, the likely values of the CIR coefficients will not be known. Hence, the initial CIR coefficient strings $\tilde{\boldsymbol{C}}_{p}^{(0)}(1)$ for $p=2, \ldots, P$ are randomly generated with the aid of real and imaginary Gaussian variables. Note that at $i=0$, the users' data has to be a known bit, since the transmitted bit sequences are differentially encoded. Based on this known data bit, the CIR coefficients for $\tilde{C}_{1}^{(0)}(1)$ can be estimated from the output of the matched filters. These estimated CIR coefficients will be interfered by the MAI and hence will not be accurate. Nevertheless, this CIR estimate will provide a good foundation for the GA to evolve from. For the CIR coefficients of subsequent signaling intervals, i.e., for $i>0$ at $y=1$, we used the CIR coefficient-strings estimated from the previous symbol interval as the initial CIR coefficient strings for the present symbol interval. More explicitly, we had $\tilde{\boldsymbol{C}}_{p}^{(i)}(y=1)=\tilde{\boldsymbol{C}}_{p}^{(i-1)}(y=Y)$ for $p=1, \ldots, P$, since the CIR coefficients are correlated between consecutive signaling intervals as governed by (4). The parameter $y=Y$ is associated with the GA generation corresponding to the termination of the search, as seen in Fig. 2.

\section{B. Evaluation}

Associated with the $p$ th combination string is a so-called figure of merit-more commonly known in GAs as the fitness value-which has to be evaluated, as seen in Fig. 2. The fitness value, denoted by $f\left[\tilde{\boldsymbol{C}}_{p}^{(i)}(y), \tilde{\boldsymbol{b}}_{p}^{(i)}(y)\right]$ for $p=1, \ldots, P$ is computed by substituting the elements of both the $p$ th CIR coefficient-string and the $p$ th data-string into the objective function or LLF of (7).

\section{Selection}

The exploitative property of the GA is derived from two GA operations referred to as selection and crossover [28]. The crossover operation will be highlighted in the next subsection. Let us refer to the elements that constitute the optimal solution as good elements. Any other elements are referred to as bad elements. For example, if the optimal solution constitutes a string containing all +1 elements, then any +1 in a string will be a good element while any -1 in the string will be a bad element. Intuitively, strings having a high fitness in the sense of (8) will contain more good elements and hence should be exploited further. At the same time, strings having a low fitness value should be discarded. Using a so-called truncated selection technique [29], as shown in Fig. 2, following the termination test, the $T$ number of fittest combination strings in the population 


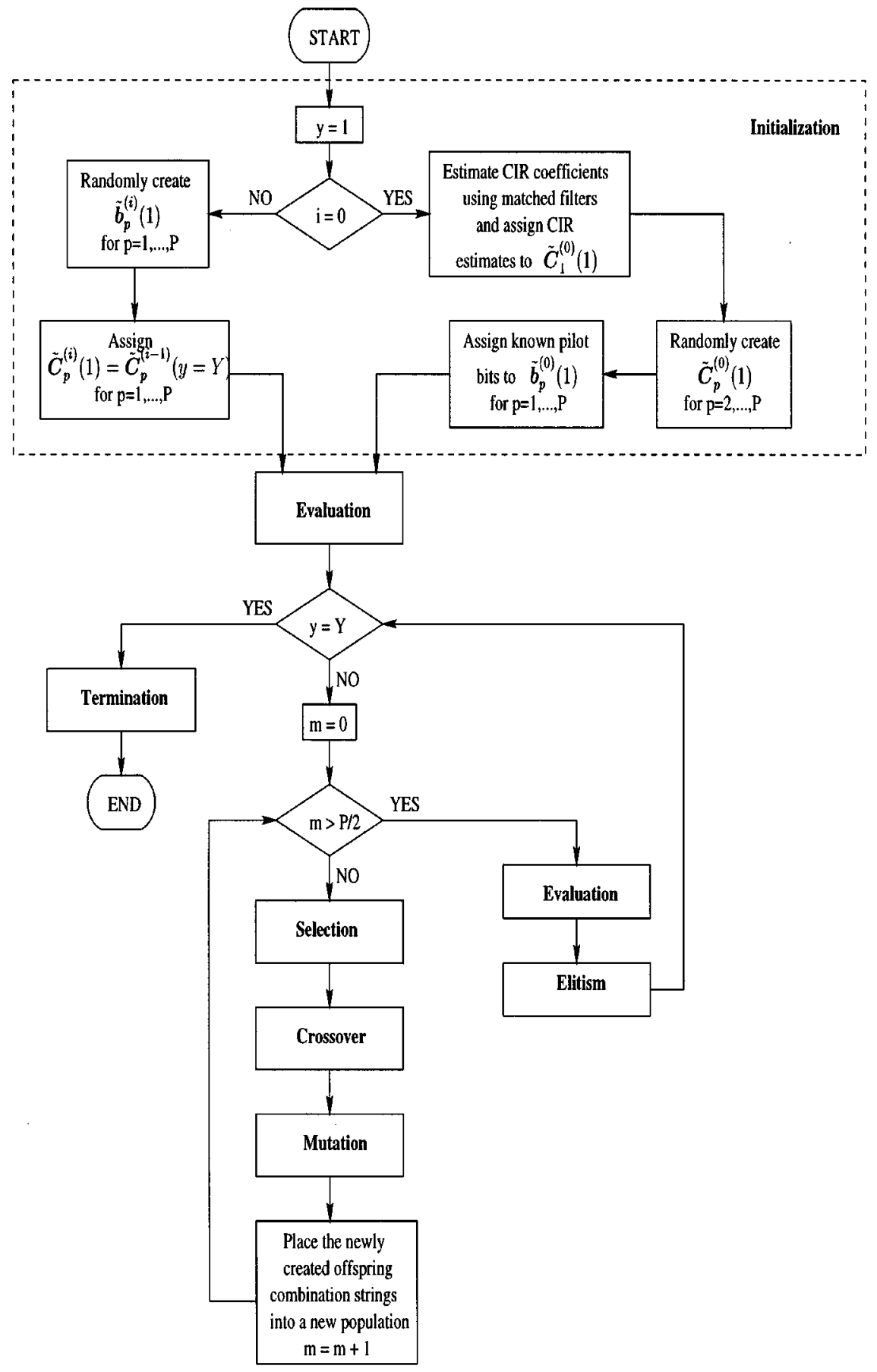

Fig. 2. Flowchart depicting the structure of the proposed genetic algorithm used to jointly estimate the users' CIR coefficients and to detect the transmitted bits at the $i$ th signaling interval.

for the generation concerned are placed in the so-called mating pool [28]. The strings in the mating pool will then be used for subsequent exploitation and exploration of the solution space. In a way, this selection technique is analogous to a tree-search type algorithm, in which only a limited number of paths are retained as survivors based on their corresponding metrics.

\section{Crossover}

The exploitation of the strings in the mating pool is carried out by the so-called crossover operation [28] of Fig. 2. Simply speaking, crossover entails the exchange of elements (good or bad) with equal probability between two different strings, the so-called parents in GA parlance, selected from the mating pool. This will produce two new strings, which are referred to as offspring. These offspring will constitute the new population of the next generation.

There are three possible outcomes from the crossover operation, as illustrated by the examples in Fig. 3. We assumed that the optimum point of a given eight-variables solution space is a string contains all +1 elements of equal contribution to the objective function, a condition which we refer to as equal weight. A so-called crossover mask [32] consisting of a sequence of randomly generated $1 \mathrm{~s}$ and $0 \mathrm{~s}$ of equal probability is invoked, 
Parents

$\begin{array}{llllllllll}1 & 1 & -1 & -1 & 1 & 1 & -1 & -1 & ==== & =\end{array} \quad \begin{array}{llllllll}1 & 1 & -1 & -1 & 1 & 1 & -1 & -1\end{array}$

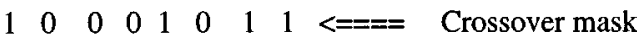

$\begin{array}{llllllllllllllllllllll}1 & -1 & 1 & 1 & 1 & -1 & -1 & -1 & ==== & = & 1 & -1 & 1 & 1 & 1 & -1 & -1 & -1\end{array}$

(a)

\section{Parents}

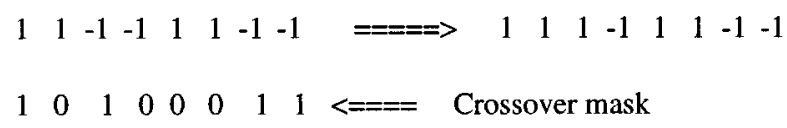

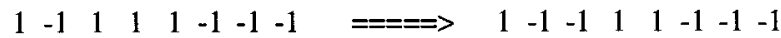

(b)

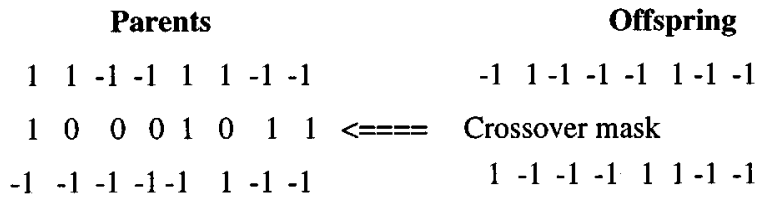

(c)

Fig. 3. Examples of crossover between two parent bit strings. (a) First outcome. No change in fitness between parents and offspring. (b) Second outcome. One offspring has one more +1 than its parents, while the other has one less +1 than its parents. (c) Third outcome. The offspring has fitness values within the range of its parents.

in which the elements are exchanged between the pair of parents at locations corresponding to a 1 in the crossover mask. In the event of the first outcome seen in Fig. 3(a), both parents exchange identical elements, resulting in identical offspring. In this case, there is no crossover gain, since the fitness of the offspring will be the same as their parents. This outcome will also occur if an even number of different elements are exchanged between parents, again assuming equal weight amongst the elements. Intuitively, this outcome will be encountered, when the population is converging toward the optimum, either global or local, when the strings are almost identical. Premature convergence toward a local optimum can be avoided by employing the so-called mutation operation, which will be highlighted later in Section III-E.

According to the second outcome, two offspring are produced, one of which has a higher fitness than both of its parents due to gaining additional good elements, as shown in the figure. On the other hand, the other offspring has a lower fitness than both of its parents due to losing good elements. For equal weight elements, this occurs when both parents exchange an odd number of different elements. This outcome is usually encountered during the first few generations of a GA when both parents have nearly equal fitness, but they are still sufficiently diverse. In this case, there is a crossover gain, since a string of higher fitness was found, which will then be evolving further in the subsequent generations.

The possible third outcome is encountered when one of the parents is a highly fit string, while the other one has a low fitness value, as shown in Fig. 3(c). They produce offspring having fitness values that lie between those of their parents. In this case, there is a crossover loss, since a highly fit string, i.e., one of the parents, is lost in the subsequent generation. In order to prevent this loss, the process of so-called elitism [29] is employed, which will be highlighted later in Section III-F.

The crossover operation is then repeated for different parents selected with equal probability from the mating pool in order to produce $P$ offspring out of which $T$ will become potential parents and in turn will produce offspring for subsequent generations. The relative occurrence frequency of the first and third outcomes largely depends on the value of $T$. If the value of $T$ is too low, certain high-fitness strings may not be evolving to future generations and the same parents are always selected, resulting in almost identical offspring (outcome one). On the other hand, if $T$ is too high, too many low-fitness strings may be surviving to future generations (outcome three). Hence, if the value of $T$ is appropriately chosen, then intuitively we can see that the crossover operation of the GA is capable of exploiting the good elements of highly fit strings in order to produce strings with even higher fitness. Based on the same concept, the data vector elements and CIR vector elements belonging to a pair of parents will be exchanged using the same crossover mask in our proposed joint symbol detector and CIR estimator.

\section{E. Mutation}

We have seen that the crossover operation can cause premature convergence, which will result in suboptimal solutions. This usually occurs when the strings in the mating pool are identical or almost identical, resulting in identical offspring. Referring to our previous example, if all the strings in the mating pool have a bad element at the same location, then further crossover processes between any of these strings will not be able to remove the bad element, since all the $P$ offspring will also "inherit" the bad element. In order to escape this trapped situation, the so-called mutation [28] operation is employed. The mutation procedure of Fig. 2 refers to the alteration of the value of each element in the offspring with a probability denoted by $p_{m}$. In the case of the data string, the mutation process simply inverts the bit value of the element concerned from 1 to -1 or vice versa. Hence, in our example, if $P$ and $p_{m}$ are sufficiently high, then the bad element in certain offspring will be mutated to a good element. If these offspring are later transferred to a mating pool and selected as parents, then this good element will be passed on to future generations.

Implementing mutation in conjunction with the complex-valued CIR coefficient-string is slightly more complicated, since it is difficult to distinguish between a good and bad element [30]. We can only strive for achieving a value that is as close to the optimum value as possible. Hence, adopting the constraint of (4) in order to search for the optimum CIR coefficients is sensible, since the previous estimated CIR coefficients will be relatively close to the optimum value of the current CIR coefficients in a slowly fading channel. When a complex-valued CIR vector element is picked for mutation, the direction of mutation is chosen randomly with equal probability for both the real and imaginary part of the complex CIR vector element. Then a real-valued mutation size $\hat{\Delta}_{k}^{(i)}(y)$ is randomly generated, whose value ranges between $\left[0, \lambda_{\max }(y)\right]$. The 
value of the real and imaginary parts of the element are then increased or decreased accordingly by a magnitude prescribed by the mutation size

$$
\begin{gathered}
\Re\left[\tilde{c}_{p, k}^{(i)}(y)\right]=\Re\left[\tilde{c}_{p, k}^{(i)}(y-1)\right] \pm \hat{\Delta}_{k}^{(i)}(y-1) \\
\Im\left[\tilde{c}_{p, k}^{(i)}(y)\right]=\Im\left[\tilde{c}_{p, k}^{(i)}(y-1)\right] \pm \hat{\Delta}_{k}^{(i)}(y-1) \\
\text { for } y=2, \ldots, Y .
\end{gathered}
$$

Notice that a limit is placed on the value of $\hat{\Delta}_{k}^{(i)}(y)$ by the parameter $\lambda_{\max }(y)$ so that the difference in phase between the mutated and original channel coefficients should be less than $\pi$. This is to ensure that the phase ambiguity will not change from the $(i-1)$ th symbol to the $i$ th symbol, unless the phase is near zero, as we shall see in the context of one of our simulation results in Section IV. On the other hand, the value of $\lambda_{\max }(y)$ should be sufficiently high-especially for high Doppler shifts-in order to track the changing CIR coefficients from one symbol to the next. In our simulations, $\lambda_{\max }(y)$ is gradually decreased over the course of the evolution according to $\lambda_{\max }(y)=\lambda_{\max }(y-1) * 0.75$, if and only if the maximum fitness of the population of the $(y-2)$ th generation is the same as that of the $(y-1)$ th generation. By gradually decreasing the mutation size, we can fine-tune the estimated CIR coefficients over the course of the evolution.

We have seen that mutation can prevent convergence to a local optimum. On the other hand, the mutation operation can also change a good element to a bad element. This is definitely undesirable, especially if the offspring, which constitutes the actual global optimum, was mutated to a suboptimum solution. On the other hand, mutation creates an opportunity to explore different areas of the solution space and hence promotes exploration. Hence, the value of $p_{m}$ must be chosen carefully in order to prevent excessive mutation. Typically $p_{m}=1 / K$ where $K$ is the number of users so that, on average, only one element per string is mutated.

\section{F. Elitism}

We have seen that the crossover operation presents a danger of losing a high-fitness string. In order to prevent the loss of these strings, we identify the string having the highest fitness in the mating pool corresponding to the previous generation and replace the lowest-fitness offspring found in the new population by it.

In the context of our proposed joint data detector and CIR estimator:

$$
\min \left\{f\left[\tilde{C}_{1}^{(i)}(1), \tilde{\boldsymbol{b}}_{1}^{(i)}(1)\right], \ldots, f\left[\tilde{C}_{P}^{(i)}(1), \tilde{\boldsymbol{b}}_{P}^{(i)}(1)\right]\right\}
$$

is replaced by

$$
\begin{aligned}
\max \left\{f\left[\tilde{\boldsymbol{C}}_{1}^{(i-1)}(Y), \tilde{\boldsymbol{b}}_{1}^{(i-1)}(Y)\right]\right. & \ldots, \\
f & {\left.\left[\tilde{\boldsymbol{C}}_{P}^{(i-1)}(Y), \tilde{\boldsymbol{b}}_{P}^{(i-1)}(Y)\right]\right\} . }
\end{aligned}
$$

Hence, the highest-merit combination string of a particular generation is propagated to the next generation. This strategy is known as elitism [29].

We can see that by employing the combination of the crossover, mutation, and elitism operations governed by probabilistic rules, the GA is capable of exploiting the good elements in highly fit strings in order to search for strings of even higher fitness, and it also ensures that the fittest will survive. At the same time, it also allows the opportunity to explore other parts of the solution space.

\section{G. Termination}

The GA can be terminated if there is no improvement in the maximum fitness value of the population after several iterations. This will ensure with a high probability that the global optimum is found at the expense of high computational complexity and long convergence time. In multiuser detection, it is more desirable to detect the data fast and at a low complexity. Hence, we terminate the GA of Fig. 2 after a specified number of $y=Y$ generations. In the context of our proposed joint data detector and CIR estimator, the combination string corresponding to the highest-fitness value is finally chosen as the estimated users' CIR coefficients and transmitted bits.

For a given population size $P$ with the GA terminated after $y=Y$ generations, the number of objective function evaluations required to detect the users' transmitted bit at each signaling interval is equal to $P \times Y$. By contrast, the computational complexity of the ML optimum detector using exhaustive search is equivalent to $2^{K}$. Hence, by adjusting the values of $P$ and $Y$, the complexity of the GA-based detector as well as its BER performance can be controlled, as we shall see in the next section. We will also show that the GA-based multiuser detector is capable of attaining a near-optimum performance with the aid of perfect CIR estimation at a significantly lower computational complexity compared to the optimum multiuser detector.

\section{Simulation Results}

In this section, our simulation results are presented in order to demonstrate the performance of the proposed detector in the context of both CIR estimation and symbol detection independently as well as jointly. A summary of the various parameters that are used in our simulations is shown in Table I. Unless otherwise specified, it was assumed that our system supported $K=10$ users with symbol synchronous transmissions over a single-path Rayleigh fading channel. The signature sequences for all users were randomly generated with a processing gain of $N_{c}=31$ and the received signal power of the users, $\xi_{k}, k=1, \ldots, K$ was assumed to be known by the receiver. The Doppler frequency and the bit rate were $f_{d}=200 \mathrm{~Hz}$ and $R_{b}=64 \mathrm{~kb} / \mathrm{s}$, respectively, for all users. Let us first evaluate the CIR estimation performance of the proposed detector, followed by its BER performance evaluation.

\section{A. Channel Estimation Performance}

Fig. 4(a) and (b) demonstrates the tracking capability of the GA-based CIR estimator both in conjunction with known bits and unknown bits, respectively. Specifically, a snapshot of the estimated real and imaginary components of the channel coefficient of a user is compared with its corresponding true value. Notice the mirror image of the estimated components after about 400 symbols with respect to the zero level of the $y$ axis in Fig. 4(b) using unknown bits. This will result in the phase ambiguity we have mentioned previously due to the $180^{\circ}$ change in the phase 
TABLE I

SUMmary OF THE VARIOUS PARAMETERS USED IN OUR SimUlations

\begin{tabular}{c|l}
\hline Symbol & Description \\
\hline System parameters \\
\hline$K$ & Number of users \\
\hline$M$ & Number of data bits per frame \\
\hline$N_{c}$ & Number of chips per bit \\
\hline$f_{d}$ & Doppler frequency \\
\hline$R_{b}$ & Data rate \\
\hline$\xi_{k}$ & Average received bit energy of the $k$ th user \\
\hline Genetic algorithm parameters \\
\hline$P$ & Population size \\
\hline$p_{m}$ & Mutation probability \\
\hline$\lambda_{m a x}(1)$ & Maximum mutation size for real-valued variables at initialization \\
\hline$T$ & Number of individuals considered for selection \\
\hline$Y$ & Number of generations per signalling interval \\
\hline
\end{tabular}

(or the sign in the case of BPSK). This change in the phase is caused by the mutation process in the attempt to estimate the desired channel coefficients. The bits in this ambiguity region will be detected in error, unless differential encoding and decoding are invoked. However, on the whole, the GA-based channel estimator was capable of tracking closely the channel variations, regardless of whether the bits were known or unknown.

In order to quantify the channel estimator's performance, the mean squared error (MSE) between the true value and the estimated value of the channel's attenuation was obtained. Fig. 5 compares the MSE performance of the GA-based channel estimator for different $\xi_{k} / N_{0}$ values and for $\lambda_{\max }(1)=0.05$ and $\lambda_{\max }(1)=0.1$ measured over a frame of 200 known bits and averaged over 200 transmitted frames with equal average received bit energy for all users. It is seen that GAs using $\lambda_{\max }(1)=0.05$ can achieve a lower MSE than in conjunction with $\lambda_{\max }(1)=0.1$. However, the former suffered from a longer convergence period.

Fig. 6 examined the effects of different $\lambda_{\max }$ (1) values on the achievable MSE for various $f_{d} T_{b}$ and $\xi_{k} / N_{0}$ parameter values. From the figure we can see that the value of $\lambda_{\max }(1)$ can have a significant impact on the achievable MSE for different $f_{d} T_{b}$ values. For example, when $f_{d} T_{b}=0.001, \lambda_{\max }(1)=0.02$ gives the optimal MSE for all $\xi_{k} / N_{0}$ values. However, for the same $\lambda_{\max }(1)=0.02$, the MSE for $f_{d} T_{b}=0.01$ becomes excessive due to the fact that a low $\lambda_{\max }(1)$ value is incapable of tracking the rapidly changing channel coefficients between symbols. Hence, in our simulations, we have adopted $\lambda_{\max }(1)=0.1$ since it exhibits a fairly consistent MSE over a $f_{d} T_{b}$ range of 0.01 to 0.001 , and it is also $\ll \pi$; hence it avoids the phase-ambiguity problem.

Fig. 7 compares the MSE of the proposed channel estimator to that of the conventional correlation-type estimator [16]. The

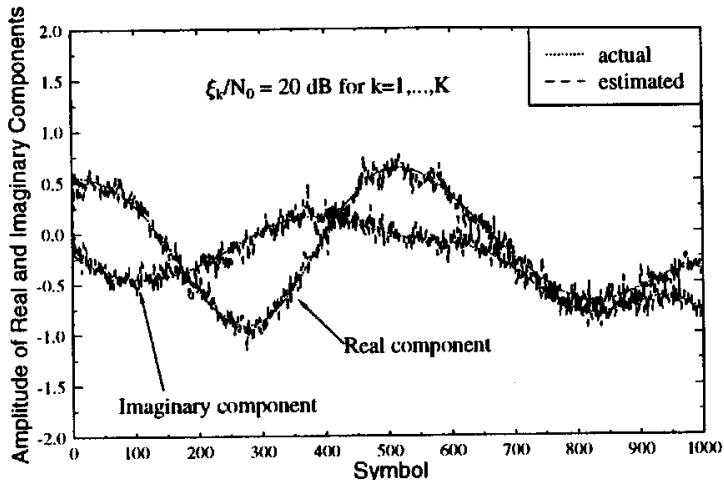

(a)

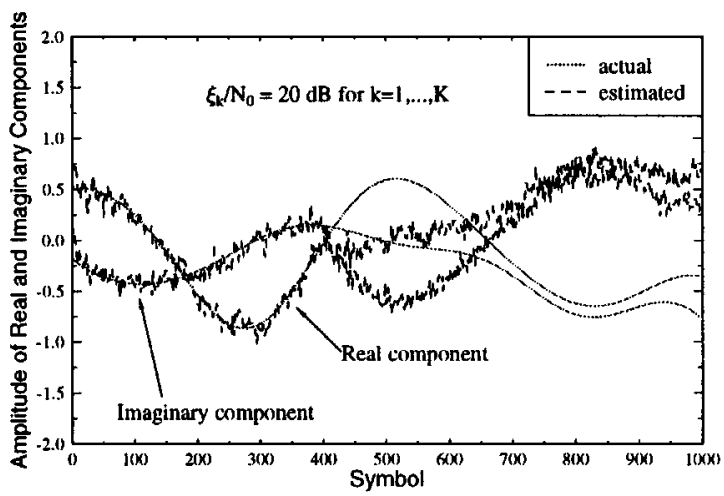

(b)

Fig. 4. Snapshot of the estimated real and imaginary components of the CIR coefficients corresponding to one user as compared to its true value for a narrowband Rayleigh-fading channel at $f_{d}=200 \mathrm{~Hz}$, where $K=10$, $P=40, Y=10, T=5, \lambda_{\max }(1)=0.1$, and $p_{m}=0.1$. (a) CIR coefficient estimation with the aid of known bits. (b) CIR coefficient estimation with the aid of unknown bits.

single-user bound using the linear minimum mean-squared error (LMMSE) channel estimator given in [33] was also plotted for comparison. It can be seen that our proposed GA-based channel estimator exhibited a significantly lower MSE value than that of the conventional estimator, and its performance was not far from the single-user bound.

\section{B. Symbol Detection Performance}

In order to evaluate the BER performance of the GA-based detector, a frame length of $M=640$ bits was employed by each user. In this subsection, the CIR coefficients are assumed to be perfectly estimated, and, hence, the results were shown in the context of nondifferential BPSK modulation where the symbols are not differentially encoded.

In order to give an impression of how the GA manages to detect the users' transmitted bits over the course of evolution given a population of randomly generated possible solutions at the beginning, the BER performance of the proposed detector at each generation is shown in Fig. 8 at $\xi_{k} / N_{0}=30 \mathrm{~dB}$ in conjunction with perfect channel estimates, i.e., when the GA was not invoked for channel estimation. As we have mentioned in Section III, the crossover operation will efficiently identify the areas in the solution space, where the optimal solution might be located. This can be seen from the steep slope during the first few generations. However, crossover alone will not find the optimal solution, 


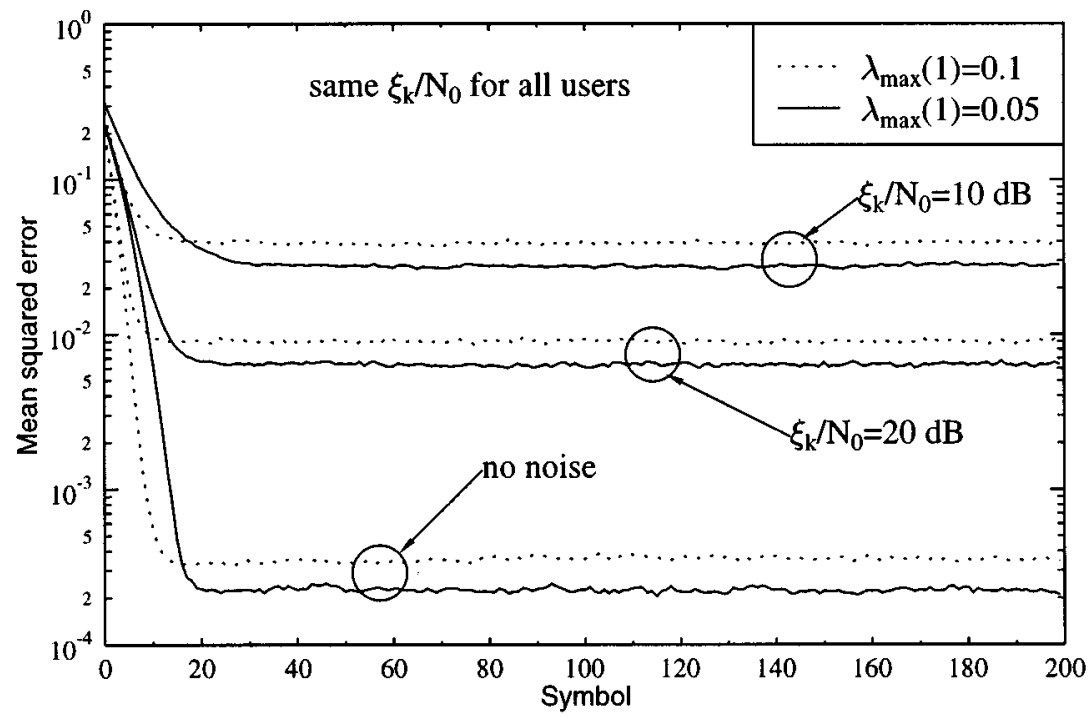

Fig. 5. Average mean squared channel estimation error in a $K=10$-user synchronous CDMA system over a frame of 200 known bits and averaged over 200 transmitted frames with equal average received bit energy for all users over a narrow-band Rayleigh channel at $f_{d}=200 \mathrm{~Hz}$, where $P=40, Y=10, T=5$, and $p_{m}=0.1$.

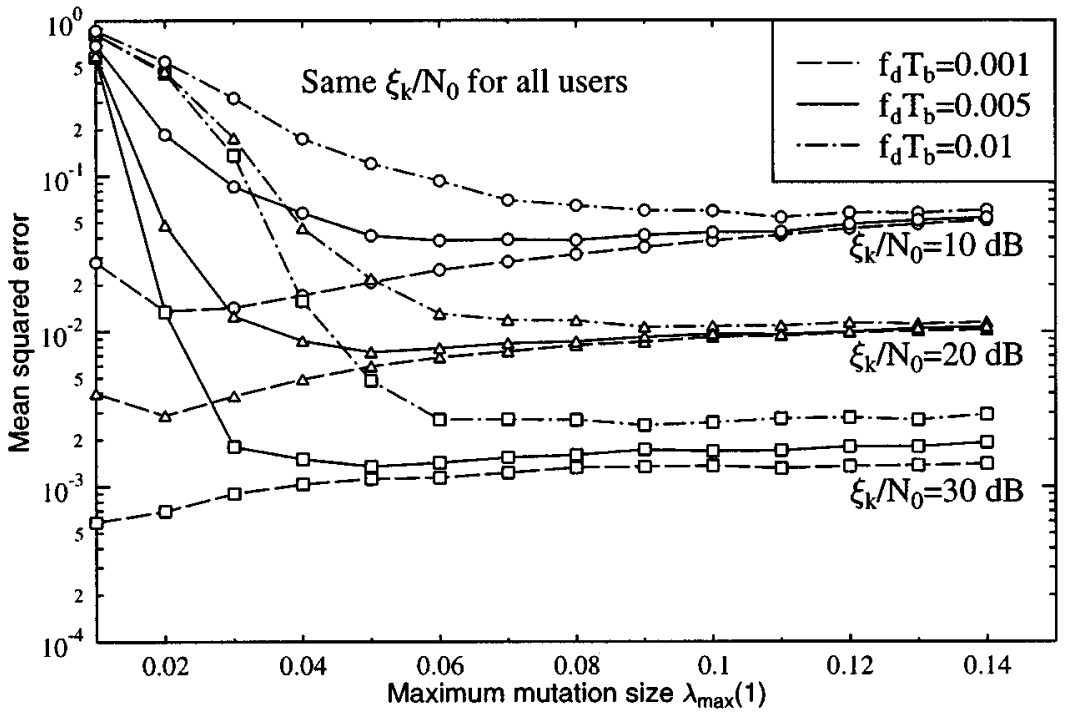

Fig. 6. Average mean squared channel estimation error after convergence in a $K=10$-user synchronous CDMA system with known bits and equal average received bit energy for all users over a narrowband Rayleigh-fading channel at various $f_{d} T_{b}$, where $P=40, Y=10, T=5$, and $p_{m}=0.1$.

when no mutation is invoked, resulting in a suboptimal solution, as shown in Fig. 8. Hence, when crossover is used in conjunction with mutation, the BER performance is improved significantly, as shown in Fig. 8. Finally, elitism is employed in order to ensure that the best string is not lost over the course of the evolution. Hence, we can see that GAs associated by elitism will always perform better than without elitism, as illustrated in Fig. 8 .

Fig. 9 characterizes the BER performance of the proposed detector in conjunction with perfect channel estimates for different number of generations $Y$ and different population sizes $P$. The single-user bound was computed using the following equation for BPSK modulation [34]

$$
P_{2}=\frac{1}{2}\left(1-\sqrt{\frac{\bar{\gamma}_{c}}{1+\bar{\gamma}_{c}}}\right)
$$

where $\bar{\gamma}_{c}=\xi_{k} / N_{0}$. As can be seen from the figure, the combination of $P=40$ bit strings and $Y=10$ generations-which constitutes $40 \times 10=400 \mathrm{LLF}$ evaluations according to (7)—was capable of achieving a near-optimum single-user-like BER performance. By contrast, the optimum multiuser detector using exhaustive search would require $2^{10}=1024$ LLF evaluations. For $\xi_{k} / N_{0}$ values beyond $40 \mathrm{~dB}$, the system exhibited an error floor due to the performance limitations of the GA in conjunction with the given $Y$ and $P$ values studied. At lower values of $Y$ and $P$, the error floor occurred at a lower $\xi_{k} / N_{0}$ value. For instance, at $Y=10$ and $P=20$, which constitutes $200 \mathrm{ob}-$ jective function evaluations according to (7), the error floor occurred at an $\xi_{k} / N_{0}$ value of about $32 \mathrm{~dB}$, while for $\xi_{k} / N_{0}$ values up to $24 \mathrm{~dB}$, the detector exhibited near-optimum BER performance. Hence, the proposed GA-based detector was capable of 


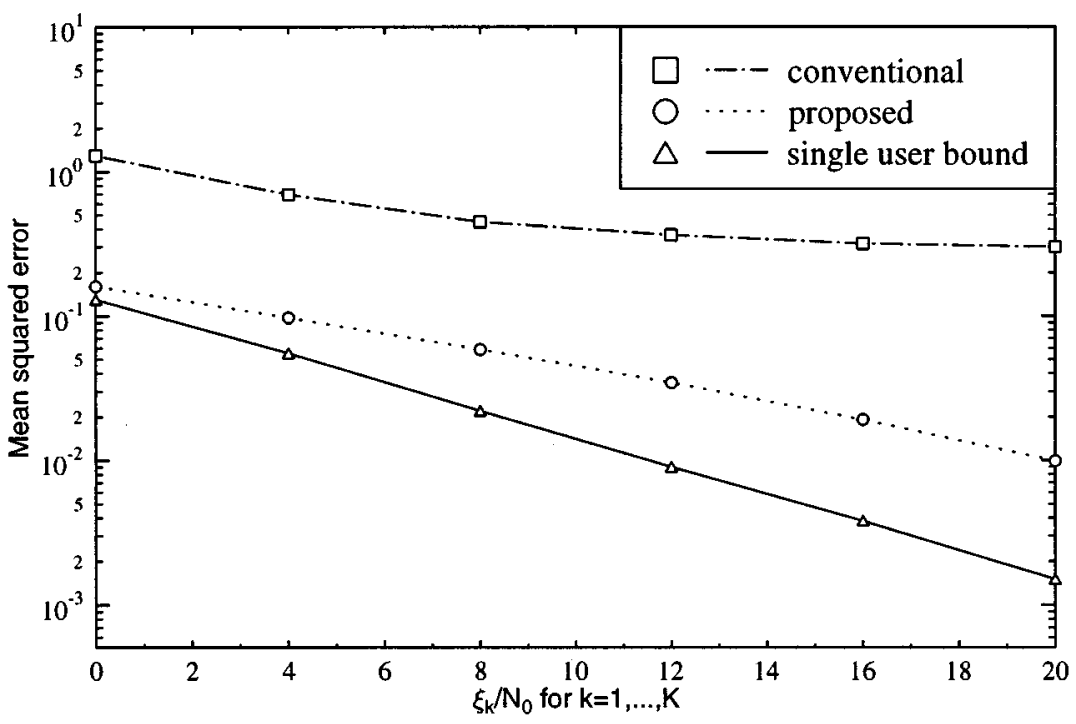

Fig. 7. Average mean squared channel estimation error in a $K=10$-user synchronous CDMA system with known bits as compared to that of a conventional correlator-type estimator in a $K=10$ user system and a single user LMMSE estimator over a narrowband Rayleigh channel at $f_{d}=200 \mathrm{~Hz}, P=40, Y=10$, $T=5, \lambda_{\max }(1)=0.1$, and $p_{m}=0.1$

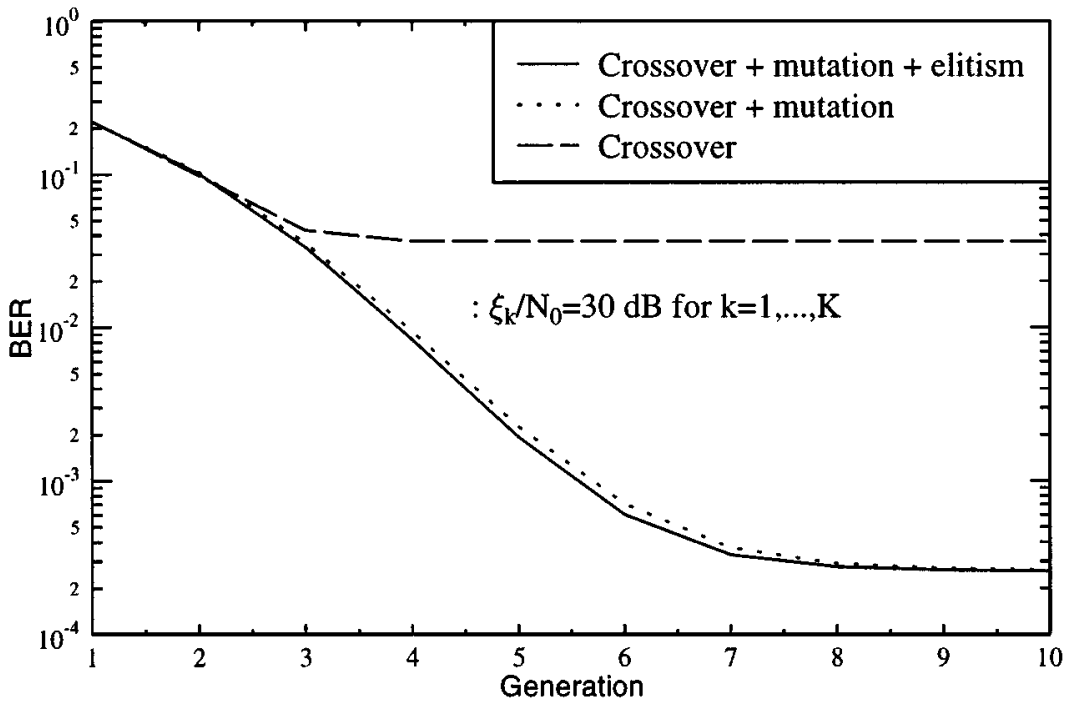

Fig. 8. BER performance of the proposed GA-based detector as a function of the number of generations assuming equal bit energy for all $K=10$ users with perfect channel estimation, where $P=40, Y=10, T=5$, and $p_{m}=0.1$.

offering a tradeoff between computational complexity and an optimum BER performance. We also note that GAs using a higher number of generations per signaling interval gave a better BER performance than GAs having a larger bit string population size $P$ and a lower number of generations $Y$ at the same computational complexity. For example, in Fig. 9 the BER performance of the $P=20, Y=10$ scenario was better than that of the $P=40, Y=5$ arrangement, both of which require $200 \mathrm{ob}-$ jective function evaluations according to (7).

In order to show that the computational complexity of the GA is not exponentially dependent on the number of users $K$, the BER performance was evaluated for various number of users, employing $P=40,80,120,160,200$ in conjunction with $Y=$ 10. The results are shown in Fig. 10. At $P=40$ and $Y=$ 10 , we can see that the BER performance gradually degrades upon increasing number of users, due to the limited population size $P$, which was too small for adequately exploring a sig- nificantly larger search space. As the population size $P$ is increased, the BER improves. With a population size of $P=160$, we can see that the GA-assisted detector is capable of attaining a near-optimal performance, when supporting $K=20$ users. More importantly, we noted that the number of LLF evaluations, denoted within the brackets in the legend of Fig. 10, increase more slowly than exponentially with the number of users. For example, when $K$ is increased from 10 to 16 , the population size $P$ has to be increased from 40 to 120 , in order to maintain the same level of performance. This constituted a factor of 1200/400 $=3$ increased computational complexity when $K$ was increased from 10 to 16, while maintaining a near-optimum BER performance. By contrast, the computational complexity of the optimum multiuser detection using exhaustive search would be increased by a factor of $2^{16} / 2^{10}=64$. Similarly, when $K$ is increased to 20, a population size of $P=160$ is sufficient for attaining the same level of BER performance. This consti- 


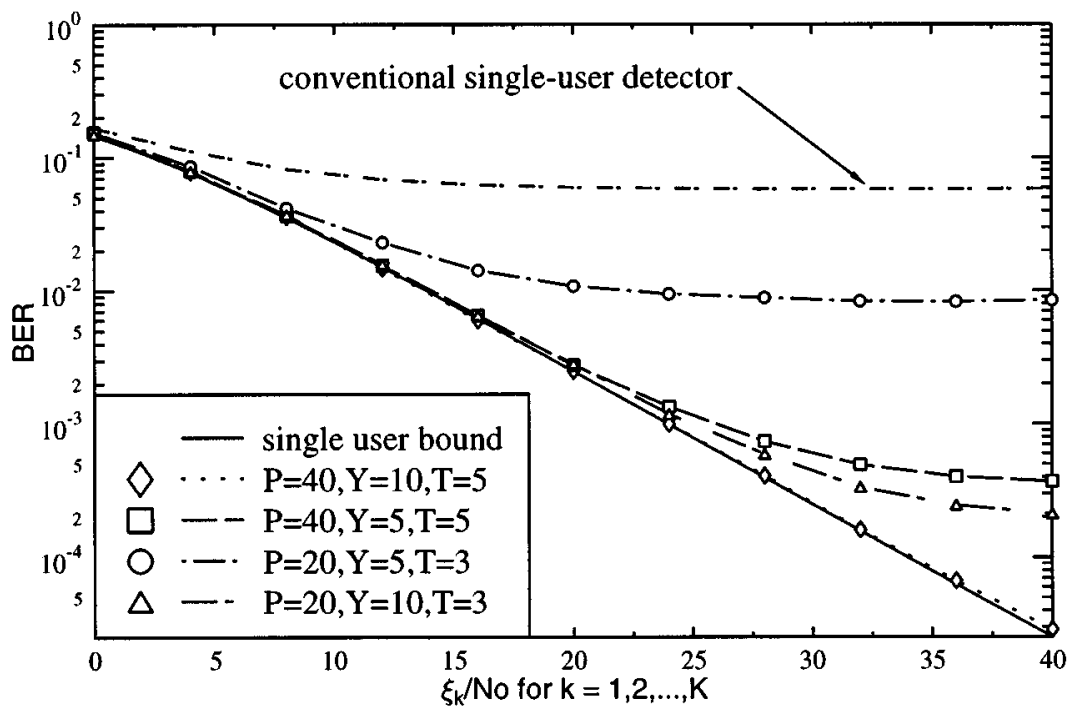

Fig. 9. BER performance of the proposed GA-based detector for $K=10$ users with various combinations of $P, Y$, and $T$ in conjunction with perfect channel estimation over narrowband Rayleigh-fading channels at $f_{d}=200 \mathrm{~Hz}$, where $\lambda_{\max }(1)=0.1$ and $p_{m}=0.1$.

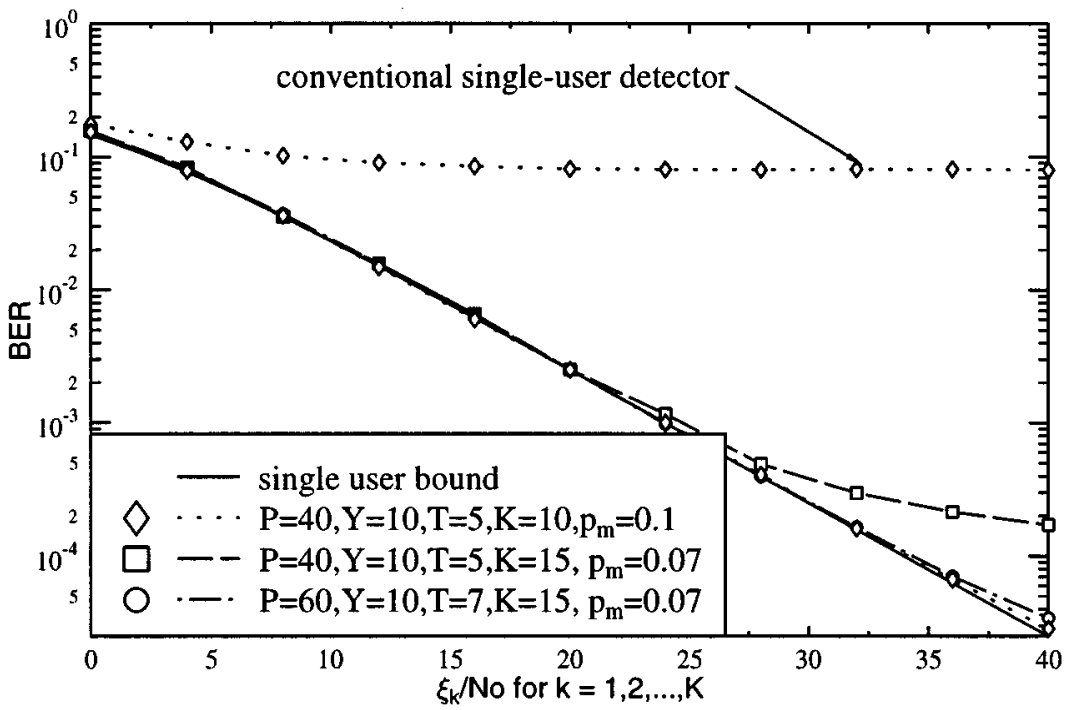

Fig. 10. BER performance of the proposed GA-based detector for as a function of the number of users $K$ with various population sizes $P$ in conjunction with perfect channel estimation over narrowband Rayleigh-fading channels at $f_{d}=200 \mathrm{~Hz}$, where $\lambda_{\max }(1)=0.1, Y=10, T=5$, and $p_{m}=0.1$.

tuted only a factor of 1600/400 $=4$ increased computational complexity. Furthermore, in contrast to the reduced tree-search type algorithms [8], [9]—which can also achieve a near-optimum BER performance at a complexity lower than that of the optimum detector-our GA-based multiuser detector does not require any memory capacity, since all information related to previous generations can be erased. Additionally, for the treesearch algorithms a noise whitening filter is required.

\section{Joint Symbol Detection and Channel Estimation Performance}

In the context of joint channel estimation and symbol detection, the bit sequence in the frame was differentially encoded with the first bit made known to the receiver. The average BER performance was evaluated after the detected bits were differentially decoded.
Fig. 11 shows the BER performance of the proposed joint channel estimator and symbol detector for processing gains of $N_{c}=31$ and $N_{c}=127$. The BER performance of the GA-based symbol detector using imperfect channel estimation having an MSE of 0.01 and 0.001 is also shown. Also plotted in the figure are the differentially coded single user bound in conjunction with perfect channel estimation, which is given by [34]:

$$
P_{2}=\frac{1}{2}\left(1-\frac{\bar{\gamma}_{c}}{1+\bar{\gamma}_{c}}\right)
$$

as well as the differentially decoded BER performance of the proposed GA-based symbol detector assisted by perfect channel estimation. As can be observed, the joint CIR and data detector exhibited an error floor due to the imperfect channel estimation, and the MSE of the CIR estimation was somewhere between 


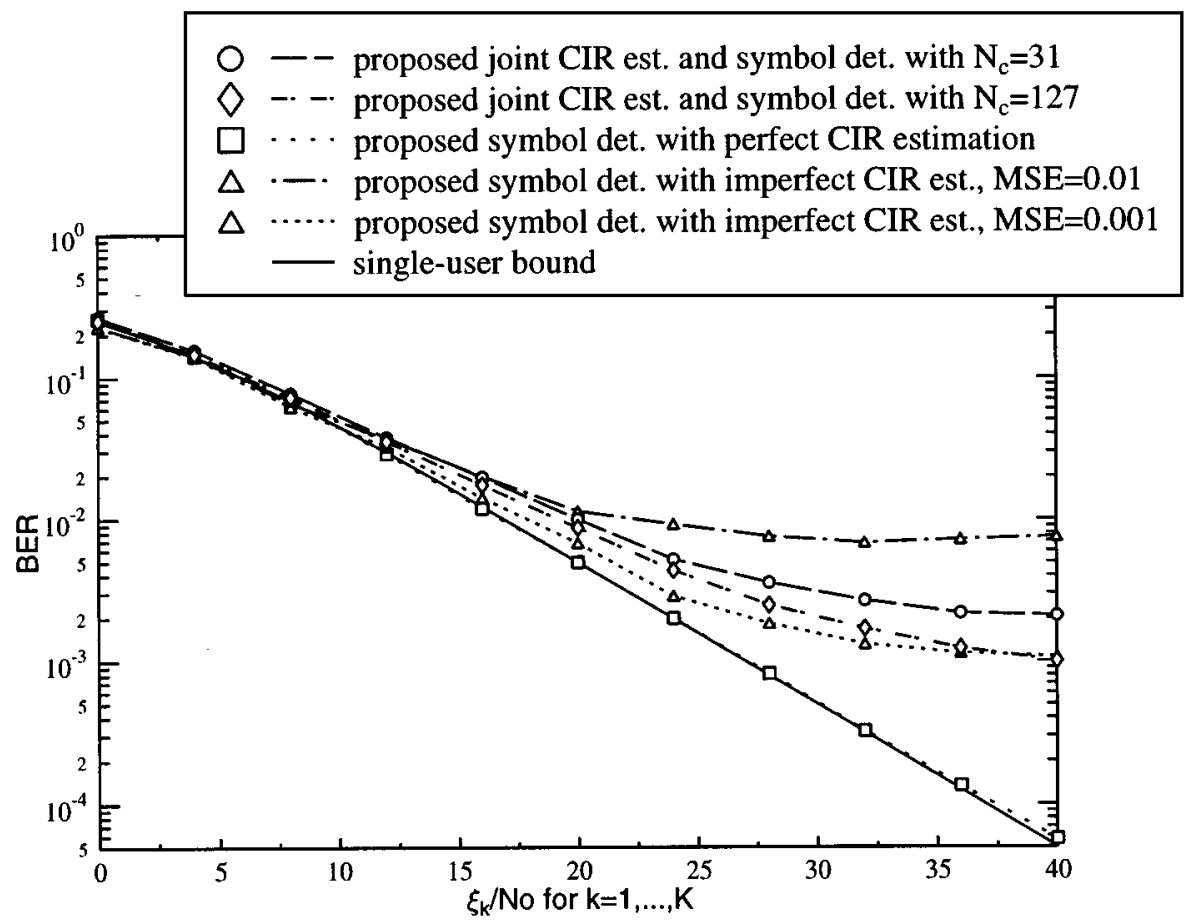

Fig. 11. BER performance of the proposed GA-based joint channel estimator and symbol detector for $K=10$ users over narrowband Rayleigh-fading channels at $f_{d}=200 \mathrm{~Hz}$ after the differential decoder, where $P=40, Y=10, T=5, \lambda_{\max }(1)=0.1$, and $p_{m}=0.1$. Results were shown for processing gains of $N_{c}=31$ and $N_{c}=127$. Also shown are the BER performances of the GA-based data detector with imperfect channel estimation with MSE values at 0.01 and 0.001 .

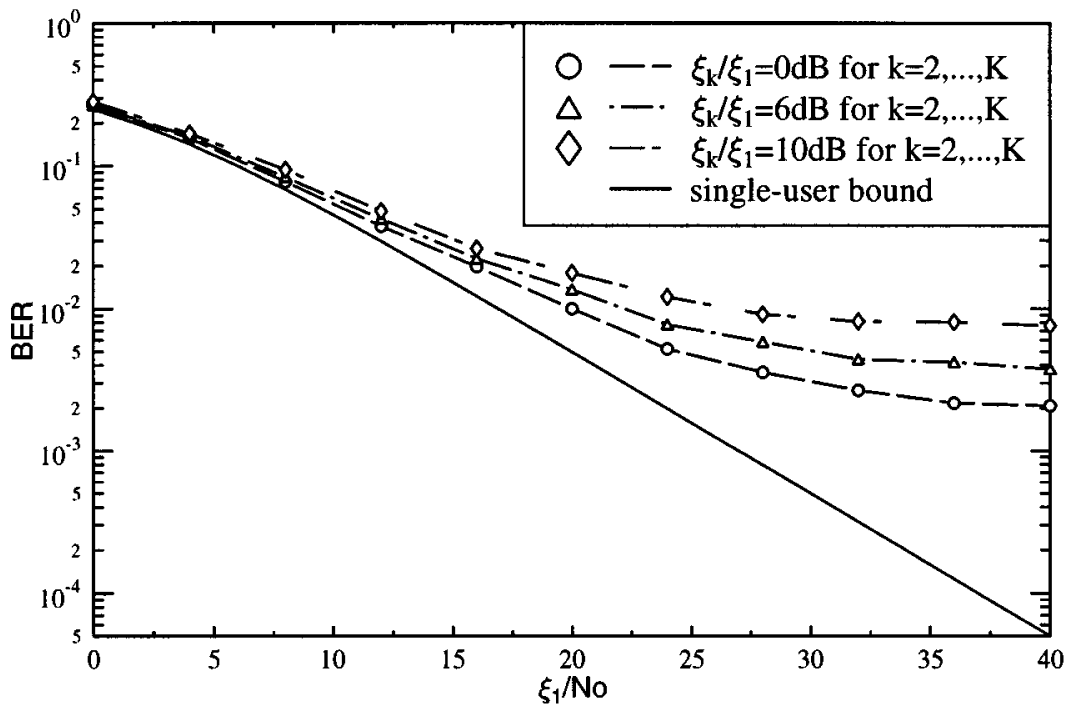

Fig. 12. BER performance of the proposed GA-based joint channel estimator and symbol detector for $K=10$ users with $\xi_{k} / \xi_{1}$ at $0 \mathrm{~dB}, 6 \mathrm{~dB}$, and $10 \mathrm{~dB}$ for $k=2, \ldots, K$ after the differential decoder of the first user, i.e., $k=1$ is the desired user, where $P=40, Y=10, T=5$, and $\lambda_{\max }(1)=0.1$.

0.01 and 0.001 . This phenomenon can also be observed in the context of other multiuser detectors. For the sake of comparison, the joint symbol detection and CIR estimation using a decorrelator and an ideal Kalman filter shown in [15] achieved a BER of $10^{-3}$ for $K=10$ users and for a processing gain of 127 . As shown in Fig. 11, our proposed detector is attaining a performance close to that. Furthermore, it should be noted that our BER is calculated over the entire length of the transmitted bit sequence, i.e., from the zeroth symbol to the $(M-1)$ th symbol, rather than after the initial convergence. Hence, the bit errors observed during the acquisition of the CIR estimates were also taken into account.

Finally, Fig. 12 characterizes the near-far resistance of the proposed joint channel estimator and symbol detector in terms of the BER of the desired user. The $x$ axis portrays the $\xi_{1} / N_{0}$ values of the desired user, while the average received bit energies of all other users were set to $0 \mathrm{~dB}, 6 \mathrm{~dB}$, and $10 \mathrm{~dB}$ higher than that of the desired user. It is seen that for both $\xi_{k} / \xi_{1}=6$ $\mathrm{dB}$ and $\xi_{k} / \xi_{1}=10 \mathrm{~dB}$, the proposed joint GA-based CIR estimator and data detector was near-far resistent up to a $\xi_{1} / N_{0}$ 
value of $16 \mathrm{~dB}$. Beyond that, a BER performance degradation was observed, eventually leading to an error floor.

\section{CONCLUSION}

In this paper, GAs were developed in order to jointly estimate the CIR coefficients as well as the transmitted bits simultaneously for all users in a symbol-synchronous CDMA system based on the ML decision rule. The system's performance was investigated using computer simulations as a 1) channel estimator with known bits, 2) symbol detector with perfect channel estimates, and 3) joint channel estimator and symbol detector.

Our results showed that as a channel estimator, the GA was capable of tracking the variations of the fading channel, while achieving a channel gain estimation MSE as low as $10^{-3}$ in a noiseless channel. The proposed symbol detector was capable of attaining a near-optimum BER performance at low $\xi_{k} / N_{0}$ values with perfect channel estimates and under the conditions of equal bit energy for all users, while maintaining a computational complexity significantly lower than that of an ML optimum multiuser detector. Upon exploiting its capabilities as a channel estimator and symbol detector, the proposed joint channel estimator and symbol detector can achieve a BER as low as $2 \times 10^{-3}$ at an $\xi_{k} / N_{0}$ value of $30 \mathrm{~dB}$ in a ten-user CDMA environment without channel coding or diversity. An error floor was observed beyond $\xi_{k} / N_{0}=30 \mathrm{~dB}$ due to the imperfect channel estimation. Furthermore, since the channel estimation and symbol detection are performed simultaneously, no pilot symbols or decision feedback are necessary, which results in a higher throughput and shorter detection time than that of explicitly trained CDMA multiuser detectors.

Based on our simulation results, we have demonstrated that the proposed GA-based multiuser detector can attain a BER performance close to the single-user bound with perfect channel estimation in the context of synchronous CDMA systems. However, further study is required in order to determine its effectiveness in asynchronous CDMA systems in conjunction with multipath propagation, which constitutes a more realistic environment. While this paper only considered the estimation of the Rayleigh fading channel gain, other forms of impairments such as imperfect power control, shadowing, and path-loss, which have a multiplicative effect on the received signal, can be estimated by the GA upon incorporating these effects in the LLF. These issues will constitute the topic of our future work. Reducing the computational complexity further by finding the optimal setup for the GA is also being investigated at present.

\section{ACKNOWLEDGMENT}

The authors are grateful for the constructive critique by the anonymous reviewers.

\section{REFERENCES}

[1] A. J. Viterbi, CDMA Principles of Spread-Spectrum Communications. Reading, MA: Addison-Wesley, 1995.

[2] R. Prasad, CDMA for Wireless Personal Communications. Norwood, MA: Artech House, 1996.

[3] S. Glisic and B. Vucetic, Spread Spectrum CDMA Systems for Wireless Communications. Norwood, MA: Artech House, 1997.
[4] T. Ojanperä and R. Prasad, Wideband CDMA for Third Generation Mobile Communications. Norwood, MA: Artech House, 1998.

[5] S. Verdú, Multiuser Detection. Cambridge, U.K.: Cambridge Univ. Press, 1998.

[6] _ - "Minimum probability of error for synchronous Gaussian multiple-access channels," IEEE Trans. Inform. Theory, vol. 32, pp. 85-96, Jan. 1986

[7] Z. Xie, C. K. Rushforth, R. T. Short, and T. K. Moon, "Joint signal detection and parameter estimation in multiuser communications," IEEE Trans. Commun., vol. 41, pp. 1208-1215, Aug. 1993.

[8] L. Wei, L. K. Rasmussen, and R. Wyrwas, "Near optimum tree-search detection schemes for bit-synchronous multiuser CDMA systems over Gaussian and two-path Rayleigh-fading channels," IEEE Trans. Commun., vol. 45, pp. 691-700, June 1997.

[9] L. K. Rasmussen, T. J. Lim, and T. M. Aulin, "Breadth-first maximum likelihood detection in multiuser CDMA," IEEE Trans. Commun., vol. 45, pp. 1176-1178, Oct. 1997.

[10] S. Abeta, S. Sampei, and N. Morinaga, "DS/CDMA coherent detection system with a suppressed pilot channel," in Proc. IEEE GLOBECOM'94, San Francisco, CA, Nov. 1994, pp. 1622-1626.

[11] J. S. Lee and L. E. Miller, CDMA Systems Engineering Handbook. Norwood, MA: Artech House, 1998.

[12] U. Fawer and B. Aazhang, "A multiuser receiver for code division multiple access communications over multipath channels," IEEE Trans. Commun., vol. 43, pp. 1556-1565, Feb./Mar./Apr. 1995.

[13] T. Kawahara and T. Matsumoto, "Joint decorrelating multiuser detection and channel estimation in asynchronous CDMA mobile communication channels," IEEE Trans. Veh. Technol., vol. 44, pp. 506-515, Aug. 1995.

[14] X. Wang and H. V. Poor, "Adaptive joint multiuser detection and channel estimation in multipath fading CDMA channels," Wireless Networks, vol. 4, no. 6, pp. 453-470, June 1998.

[15] Z. Zvonar and M. Stojanovic, "Performance of antenna diversity multiuser receivers in CDMA channels with imperfect fading estimation," Wireless Personal Commun., vol. 3, no. 1/2, pp. 91-110, 1996.

[16] T. J. Lim, L. K. Rasmussen, and H. Sugimoto, "An asynchronous multiuser CDMA detector based on the Kalman filter," IEEE J. Select. Areas Commun., vol. 16, pp. 1711-1722, Dec. 1998.

[17] M. J. Juntti, T. Schlosser, and J. O. Lilleberg, "Genetic algorithms for multiuser detection in synchronous CDMA," in Proc. IEEE Int. Symp. Information Theory, 1997, p. 492.

[18] X. F. Wang, W. S. Lu, and A. Antoniou, "A genetic algorithm-based multiuser detector for multiple-access communications," in Proc. IEEE Int. Symp. Circuits and System-ISCAS'98, vol. 4, 1998, pp. 534-537.

[19] C. Ergün and K. Hacioglu, "Application of a genetic algorithm to multistage detection in CDMA systems," in Proc. Mediterranean Electrotechnical Conf., MELECON, vol. 2, 1998, pp. 846-850.

[20] K. Yen and L. Hanzo, "Hybrid genetic algorithm based multiuser detection schemes for synchronous CDMA systems," in Proc. Vehicular Technology Conf., Tokyo, Japan, 2000, pp. 1400-1404.

[21] S. Chen and Y. Wu, "Maximum likelihood joint channel and data estimation using genetic algorithms," IEEE Trans. Signal Processing, vol. 46, pp. 1469-1473, May 1998.

[22] H.-Y. Wu and A. Duel-Hallen, "Multiuser detection with differentially encoded data for mismatched flat Rayleigh fading CDMA channels," in Proc. Conf. Information Sciences Systems, Princeton, NJ, Mar. 1996, pp. 332-337.

[23] J. Panicker and S. Kumar, "Effect of system imperfections on BER performance of a CDMA receiver with multipath diversity combining," IEEE Trans. Veh. Technol., vol. 45, pp. 622-630, Nov. 1996.

[24] D. N. Kalofonos, M. Stojanovic, and J. G. Proakis, "Analysis of the impact of channel estimation errors on the performance of a MC-CDMA system in a Rayleigh fading channel," in Proc. IEEE Global Telecommunications Conf., vol. 4, 1997, pp. 213-217.

[25] M. Stojanovic and Z. Zvonar, "Performance of multiuser detection with adaptive channel estimation," IEEE Trans. Commun., vol. 47, pp. 1129-1132, Aug. 1999.

[26] J.-M. Renders and S. P. Flasse, "Hybrid methods using genetic algorithms for global optimization," IEEE Trans. Systems, Man, Cybernetics-Part B: Cybernetics, vol. 26, pp. 243-258, Apr. 1996.

[27] J. H. Holland, Adaptation in Natural and Artificial Systems. Ann Arbor, MI: Univ. Michigan Press, 1975.

[28] D. E. Goldberg, Genetic Algorithms in Search, Optimization, and Machine Learning. Reading, MA: Addison-Wesley, 1989.

[29] M. Mitchell, An Introduction to Genetic Algorithms. Cambridge, MA: MIT Press, 1996.

[30] A. H. Wright, "Genetic algorithms for real parameter optimization," in Foundations of Genetic Algorithms, G. Rawlins, Ed. San Mateo, CA: Morgan Kaufmann, 1991, pp. 205-218. 
[31] C. Z. Janikow and Z. Michalewicz, "An experimental comparison of binary and floating point representations in genetic algorithms," in Proc. 4th Int. Conf. Genetic Algorithms, San Mateo, CA, 1991, pp. 31-36.

[32] G. Syswerda, "Uniform crossover in genetic algorithms," in Proc. 3rd Int. Conf. Genetic Algorithms, J. D. Schaffer, Ed. San Mateo, CA: Morgan Kaufmann, 1989, pp. 2-9.

[33] M. Juntti, "Multiuser demodulation for DS-CDMA systems in fading channels," Ph.D. dissertation, Univ. Oulu, Oulu, Finland, Sept. 1997.

[34] J. G. Proakis, Digital Communications, 3rd ed. New York: McGrawHill, 1995.

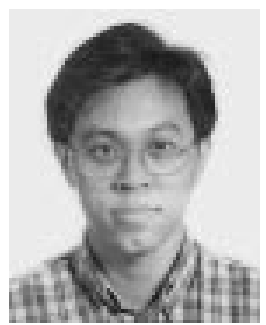

Kai Yen was born in Singapore in 1969. He received the B.Eng. degree with honors in electrical and electronics engineering from the Nanyang Technological University, Singapore, in 1996. He recently received the Ph.D. degree from the University of Southampton, U.K., funded by a scholarship from the Centre for Wireless Communications (CWC).

From 1996 to 1997, he was with CWC, National University of Singapore as a Research Engineer. His research interests include multiuser detection and modem techniques for CDMA.

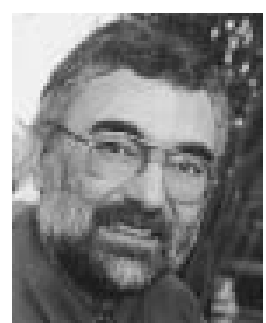

Lajos Hanzo (M'91-SM'92) received the degree in electronics in 1976 and the Ph.D. degree in 1983, both from the Technical University of Budapest, Hungary.

During his 24-year career in telecommunications, he has held various research and academic posts in Hungary, Germany, and the U.K. Since 1986, he has been with the Department of Electronics and Computer Science, University of Southampton, U.K., and has been a Consultant with Multiple Access Communications Ltd., Southampton, U.K. Currently, he holds a chair in telecommunications. He coauthored five books on mobile radio communications, published about 300 research papers, organized and chaired conference sessions, presented overview lectures, and was awarded a number of distinctions. Currently, he is managing an academic research team, working on a range of research projects in the field of wireless multimedia communications sponsored both by industry and under the auspices of the Engineering and Physical Sciences Research Council (EPSRC), U.K., the European IST Programme, and the Mobile Virtual Centre of Excellence (VCE), U.K. 